\title{
Transplantation of a 3D Bioprinted Patch in a Murine Model of Myocardial Infarction
}

\author{
Christopher D. Roche ${ }^{1,2,3,4}$, Carmine Gentile $e^{1,2,3}$ \\ ${ }^{1}$ The University of Sydney ${ }^{2}$ University of Technology Sydney (UTS) ${ }^{3}$ The Royal North Shore Hospital ${ }^{4}$ University Hospital of Wales
}

\section{Corresponding Author}

Carmine Gentile

Carmine.Gentile@uts.edu.au

\section{Citation}

Roche, C.D., Gentile, C. Transplantation of a 3D Bioprinted Patch in a Murine Model of Myocardial Infarction. J. Vis. Exp. (163), e61675, doi:10.3791/61675 (2020).

\section{Date Published}

September 26, 2020

DOI

$10.3791 / 61675$

URL

jove.com/video/61675

\section{Abstract}

Testing regenerative properties of 3D bioprinted cardiac patches in vivo using murine models of heart failure via permanent left anterior descending (LAD) ligation is a challenging procedure and has a high mortality rate due to its nature. We developed a method to consistently transplant bioprinted patches of cells and hydrogels onto the epicardium of an infarcted mouse heart to test their regenerative properties in a robust and feasible way. First, a deeply anesthetized mouse is carefully intubated and ventilated. Following left lateral thoracotomy (surgical opening of the chest), the exposed $L A D$ is permanently ligated and the bioprinted patch transplanted onto the epicardium. The mouse quickly recovers from the procedure after chest closure. The advantages of this robust and quick approach include a predicted 28-day mortality rate of up to $30 \%$ (lower than the $44 \%$ reported by other studies using a similar model of permanent LAD ligation in mice). Moreover, the approach described in this protocol is versatile and could be adapted to test bioprinted patches using different cell types or hydrogels where high numbers of animals are needed to optimally power studies. Overall, we present this as an advantageous approach which may change preclinical testing in future studies for the field of cardiac regeneration and tissue engineering.

\section{Introduction}

A heart transplant is the gold standard treatment for patients with end-stage heart failure but there is a shortage of donor organs. It requires immune system suppression to prevent graft rejection and the one-year mortality rate is $15 \%$ worldwide ${ }^{1}$. Therefore, there is a longstanding incentive to regenerate the myocardium in preclinical animal models with a view to translating to human trials $2,3,4,5,6,7,8,9$. Recent advances in 3D bioprinting of stem cells or stem cell- derived cardiac cells have gained attention as a promising approach to regenerate the myocardium ${ }^{2}, 3,9,10,11,12$.

The first human safety trials applying patches to regenerate the heart have been reported, with autologous bone marrow mononuclear cells suspended in collagen or embryonic stem cell-derived cardiac progenitor cells in fibrin, transplanted to the epicardium ${ }^{7}, 8,13$. However, for a more precise, scalable, automatable and reproducible method, 3D 
bioprinting of optimized hydrogel patches to be applied to the epicardial surface of the heart is a promising approach to regenerate the myocardium for patients who would otherwise need a heart transplant ${ }^{2}, 10,11,12$.

Before translation to human trials can occur, preclinical animal studies are needed. Preclinical in vivo models pursuing regeneration of the myocardium have been reported in pigs ${ }^{5}$, sheep ${ }^{14}$, rats ${ }^{6}$ and mice ${ }^{4}$. A common model of myocardial infarction (MI) in mice uses permanent ligation of the left anterior descending (LAD) coronary artery ${ }^{15,16}$. Among the different strains of mice used, permanent LAD ligation in C57BL6 mice has an acceptable survival rate and typically presents consistent remodeling and cardiac changes after $\mathrm{MI}^{16}$. In rodent models, several approaches have been described where cardiac tissue has been applied to the heart in pursuit of effective regeneration of damaged myocardium $4,6,17$. While large animals still represent a more clinically relevant model to test cardiac regenerative properties $^{5,14}$, the versatility and feasibility of the mouse model lends itself to this fast-moving area of study. This may avoid some of the pitfalls typical of large animal studies, including (but not limited to): 1) high animal mortality (unless diagonal coronary arteries are ligated leading to unpredictable segmental infarcts ${ }^{14}$, or the distal end of the LAD is occluded followed by reperfusion instead of permanent ligation ${ }^{5}$ ); 2) ethical issues with the relatively increased harm caused by large animal protocols compared to mice $\left.{ }^{18} ; 3\right)$ increased cost and/or feasibility issues, for instance the relative unavailability of large animal equipment such as MRI scanners ${ }^{14}$. It is also important to consider that given the extensive duration and commitment typical of large animal studies, they have the potential to become outdated before they are finished, especially with the rapid developments typical of this field. For instance, it is only recently that the critical role played by inflammatory cells and mediators in regulating cardiac regeneration has emerged $^{19,20}$. Furthermore, the critical role of preclinical studies, such as small animal models, has been highlighted by a Lancet Commission as an essential step to gain robust knowledge before moving to human trials ${ }^{21}$.

To facilitate progress in understanding mechanisms and optimizing conditions for patch-based cardiac regeneration approaches in vivo, we present a novel approach describing a 'scoop and drape' method to apply a 3D bioprinted alginate/ gelatine hydrogel patch to the surface of infarcted hearts in C57BL6 mice. The aim of this approach is to provide a versatile in vivo model to test $3 \mathrm{D}$ bioprinted patches that are likely to be feasible in broad research contexts for the rapidly-evolving field of cardiac regeneration ${ }^{2}$. This method could be adapted to test patches generated by non-bioprinting methods, different hydrogels and autologous or allogenic stem cell-derived cells within patches in vivo. However, detailed consideration of bioprinting, hydrogels or cell types is beyond the scope of this study which focuses on the surgical transplantation method.

The advantages of the protocol include that the myocardial infarction and application of a bioprinted patch are performed in one surgical procedure that can be performed quickly, with readily-available, cost effective laboratory tools and with a relatively low mortality rate. It also typically allows for a higher number of animals than large animal models in a smaller space, which permits robust comparison of multiple experimental groups, particularly useful for multiple group comparison in vivo. On the other hand, this protocol has the disadvantages that: 1) the mouse model is more distant from human heart size, anatomy and physiology than in large animal models and it does not directly translate into humans; 
2) the murine LAD branches proximally, with significant variability between individual mice, which leads to infarct size variability (a problem shared with large animal models); 3) the patch must be applied over the whole anterior heart surface, which is less precise than applying over a specific infarct area; and 4) the patch is applied immediately at the time of MI (for human use it is likely to be more clinically useful to develop a patch for application to the chronically infarcted failing heart months following the initial $\mathrm{MI}^{14}$ ).

Nonetheless, if chosen appropriately according to the hypothesis being tested, this protocol can provide critical in vivo data quickly, with high $\mathrm{n}$ numbers, in a way that is consistent with the materials, budget and expertise available in most laboratories. Compared to large animal models, it is an in vivo model that is versatile enough to adapt to emerging 3D bioprinting technologies (for example by the relative ease of performing pilot studies to test feasibility and safety before moving to larger animal models). It would be well-suited for researchers who want to generate in vivo data efficiently and inexpensively, perhaps running multiple comparisons of 3D bioprinted patches with different bioprinting parameters, cells or hydrogels in the patches. It would be especially useful for testing the interactions of different mixtures of stem cells and stem cell-derived cells with hydrogels in vivo without excess wastage of expensive cell lineages or other materials that might occur if using large scale patches. Using a mouse model would also facilitate testing of patches containing species-compatible mouse-derived cell and stem cell lineages or human-derived cells where uniform mice with a specific immune deficiency are desirable. Additionally, testing in genetically modified mouse strains could allow researchers to isolate the effects of specific genes on signaling pathways and in specific cell types relevant to cardiovascular disease, which would not currently be possible in a large animal model.

\section{Protocol}

All procedures described in this experiment were approved by the Animal Ethics Committee at the Northern Sydney Local Health District, NSW, Australia (project number RESP17/55).

\section{Anesthesia and intubation}

NOTE: Turn on and set up the stereomicroscope, the heat pad (covered with an absorbant sheet) and ventilator system.

1. Clean gloves, the surgical area, and the tools with $70 \%$ ethanol.

2. Weigh the mouse to calculate the dosage of anesthesia injected by the intraperitoneal route (ketamine $40 \mathrm{mg} /$ $\mathrm{kg}$, xylazine $5 \mathrm{mg} / \mathrm{kg}$, atropine $0.15 \mathrm{mg} / \mathrm{kg}$ ) and give the injection.

3. Once the mouse reaches a deep plane of anesthesia, shave the ventral left side of the thorax with a trimmer.

4. Place the mouse in a chamber containing $2 \%$ isoflurane (ensuring adequate extraction ventilation in the room).

NOTE: The relatively low dose of ketamine/xylazine injection together with $2 \%$ isoflurane inhalation reduces the risk of mouse death while allowing optimal intubation without waking the mouse up.

5. Place the mouse supine and restrain it from its upper incisor teeth with a 3.0 suture taped to the bench, as shown in the video. Confirm sedation by performing a toe pinch. Position a high-intensity illuminator above the mouse neck so that the oropharynx can be visualized.

NOTE: Alternatively, the mouse can be placed on the stand from the intubation kit (e.g., Kent Mouse Intubation 
Kit) with an elastic band secured under the top incisors to hold the mouth open to identify the trachea.

6. Use a curved spatula to open the jaw and another pair of spatulas/blunt forceps to lift the tongue gently out of the way. Be sure to intubate while positioned at or slightly below eye-level with the body of the mouse.

7. Visualize the opening and closing of the vocal cords. When open, insert the $20 \mathrm{G}$ plastic catheter supplied with the intubation kit.

8. Carefully transfer the intubated mouse to an operating surface equipped with a heating pad. Connect the mouse to the ventilator (e.g., MouseVent) that automatically sets the target volume based on mouse weight.

9. Deliver $1.5-2 \%$ isoflurane with oxygen (which is automatically regulated by the ventilator: ensure there is a connection from an oxygen cylinder to the automatic ventilator at $1-2 \mathrm{~L} / \mathrm{min}$ flow rate to the ventilator). Verify the intubation by checking for bilateral chest rise. Verify anesthesia by performing a toe pinch.

10. Apply opthalmic ointment (e.g., Puralube Vet Opthalmic Ointment) to both eyes to prevent them from drying out.

\section{Preparing the surgical field}

1. Secure the intubation tube with tape at the connecting site between the ventilator and the breathing tube/catheter.

2. Cut a longer piece of tape and secure its left front foot to the operating surface in a slightly elevated position. Also tape down the other extremities.

3. Clean the chest with sterile $70 \%$ isopropanol and povidone iodine solution, cleaning in a circular motion moving from center to periphery.

4. Verify anesthesia once more with a toe pinch.
5. Administer $0.08 \mathrm{mg} / \mathrm{kg}$ Temvet (buprenorphine) in $0.1 \mathrm{~mL}$ of $0.9 \%$ saline via subcutaneous injection.

\section{Left lateral thoracotomy}

1. Use fine-tip forceps to gently lift the skin at a point approximately $5 \mathrm{~mm}$ to the left of the prominent xiphoid cartilage. Use surgical scissors to create a superomedial incision in the skin from this point upwards and towards the midline, to the level of the manubrium.

2. Use curved forceps to gently separate the skin and muscle layers. Open the muscle layer, following the skin incision.

3. Identify and make an incision in the third intercostal space, following the natural angle of the ribcage.

4. Use a retractor to gently spread apart the 3 rd and 4th ribs.

5. Gently remove the thin pericardium with forceps.

6. If the LAD is not visualized, gently push the left auricle (see Supplementary Figure 1) upwards and locate the coronary arteries underneath.

\section{Left anterior descending (LAD) permanent coronary artery ligation}

1. Cut a $\sim 3 \mathrm{~mm}$ long $3-0$ silk suture and put this reinforcing 3-0 silk suture piece on top of the LAD in the same direction as the LAD (as shown in the video at time point $02: 12-02: 20)$.

2. Identify the LAD and pass a 7-0 silk suture under the LAD. If the LAD is not clearly visualized, insert the needle $1 \mathrm{~mm}$ inferior and medial to the inferiormost point reached by the tip of the left auricle during dynamic movement of the heart.

NOTE: This structure is a lighter color red to the ventricular chambers of the heart but darker than the 
adjacent lung and is best visualized in the video at time point 01:54 - 01:55 where it is visible just inferior to the superior arm of the retractor, superior to the left lung (see Supplementary Figure 1 for annotated video still image).

3. Complete two throws with the 7-0 silk suture and close it tightly passing on top of the supportive 3-0 silk suture to secure the $L A D$. If the ligation is successful, the anterior ventricular area distal from the ligature will blanch.

4. Complete the knot with a third throw in the opposite direction to secure it, ensuring no upward traction force is transmitted to the suture. Additional throws are not required to reduce the risk of damage to the myocardium or LAD by suture cutting through.

\section{Transplantation of the bioprinted patch onto the epicardium}

1. Carefully move the bioprinted patch from a six well plate to the infarct area by using the sterile inside surface of an opened surgical scalpel packet.

2. Carefully position the bioprinted patch onto the anterior epicardial surface, where it should cover the whole surface and drape over the inferior and lateral edges, covering the left ventricle and the infarct zone (blanched area).

3. Gently close and remove the retractor without directing sharp edges towards the heart.

4. Use 6-0 prolene sutures in a simple interrupted pattern to close the ribcage and the muscle layers.

5. With the Sigh Breath function while closing the chest with the 6-0 prolene sutures, inflate the lungs to remove excess air in the pleural cavity, which would otherwise become trapped in the chest cavity and result in a pneumothorax.
6. Ensure that the chest is tightly sealed.

7. Decrease the isoflurane to $1.0 \%$. Close the skin with $6-0$ prolene sutures in a simple interrupted pattern. Turn the isoflurane vaporizer off.

\section{Mouse recovery}

1. Topically apply $2 \mathrm{mg} / \mathrm{mL}$ bupivacaine in $0.9 \%$ saline to the incision. Administer also: i) Antisedan (atipamezole) 1 $\mathrm{mg} / \mathrm{kg}$; ii) Lasix (furosemide) $8 \mathrm{mg} / \mathrm{kg}$; iii) $600 \mu \mathrm{L}$ of $0.9 \%$ saline solution via a subcutaneous injection.

NOTE: Antisedan is to reverse the anesthetic more rapidly; furosemide is to offload excess fluid due to cardiac output compromise and additional fluid administered with drug injections.

2. Monitor the mouse and wait until independent breathing is observed to remove the mouse from the intubation tube.

3. When the mouse demonstrates an adequate bilateral breathing rate and depth and responds to a toe pinch, place the mouse in a clean recovery cage placed on a heat pad.

4. Provide the mouse with moist food (moistened for chewability), a water bottle and nutrient/hydrating gel. Monitor for an exaggerated breathing effort, excessive bleeding, or other potentially life-threatening complications.

5. For the next three days, administer $0.08 \mathrm{mg} / \mathrm{kg}$ Temvet (buprenorphine) in $0.1 \mathrm{~mL}$ of $0.9 \%$ saline via subcutaneous or intraperitoneal injection, twice daily, then once daily up to the fifth day following the procedure.

6. House mice in pairs separated by cage dividers to prevent isolation whilst preventing fighting behaviors. Monitor mice at least daily until the end of the experiments $(28$ 
days) with close attention to their wellbeing and increased frequency of monitoring if there are any concerns.

\section{Representative Results}

At transplantation, the viscosity of the patch at room temperature (without additional crosslinker being applied) allowed it to 'drape' over the contours of the heart (Figure 1) and move dynamically with the cardiac cycle. After the surgery, we left the patches for 28 days in vivo as studies have found this to be a suitable time period allowing for patch effects on host cardiac function ${ }^{3,4}$ (although it has been reported that full functional effects may not be seen until three months after transplantation) ${ }^{22}$. The photograph of a patch shown in situ on a mouse heart in Figure 1 was taken immediately after application, showing the ability of the patch to drape over the heart at transplantation. This representative result shows that the hydrogel allows for the patch to mold to the contours of the heart and where excessive tension occurred the hydrogel was able to split as shown by the bare (hydrogel-free) triangular area in Figure 1 (indicated by a black star in the image). Survival data (Kaplan-Meier survival curves) are shown in Figure 2 compared to mice undergoing a sham procedure (passage of a needle and suture under the LAD without ligation followed by closure of the mouse chest). 


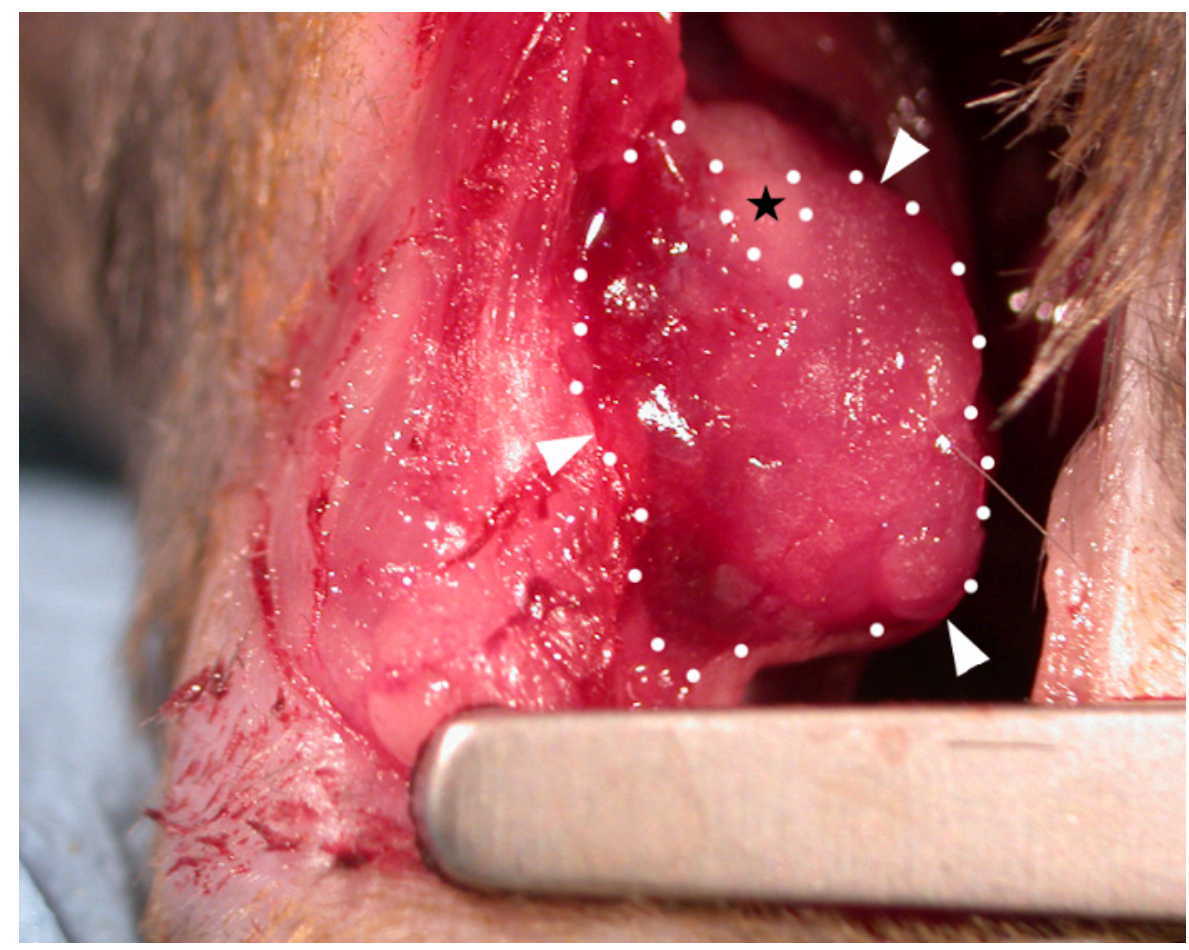

Figure 1: A bioprinted cardiac patch applied onto the epicardium of a C57BL6 mouse heart. A $10 \mathrm{~mm} \times 10 \mathrm{~mm} \times 0.4$ $\mathrm{mm}$ bioprinted patch (immediately after transplantation) containing hydrogel (alginate $4 \%(\mathrm{w} / \mathrm{v}) / \mathrm{gelatine} 8 \%(\mathrm{w} / \mathrm{v})$ in media) is shown draped over the infarcted area and adhering to the epicardial surface (white arrowheads and dotted lines = border of the patch). The patch viscosity allows it to mold to the contours of the heart and where excessive tension occurred at the superior aspect the patch has split to make a triangular bare area not covered by hydrogel (black star). Please click here to view a larger version of this figure. 


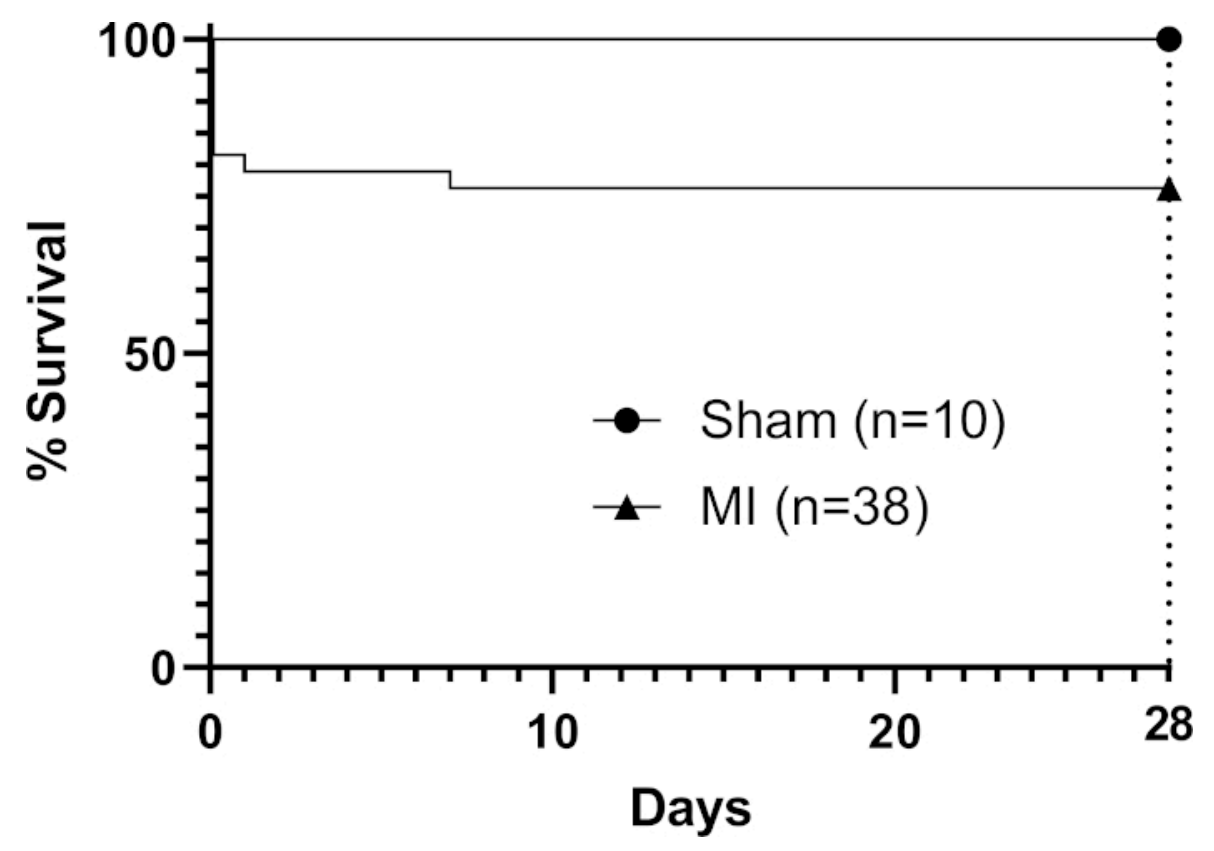

Figure 2: Kaplan-Meier survival analysis through 28 days post-MI. Nine mice in the procedural group died $(n=38)$ to give an overall mortality rate of $24 \%$. Please click here to view a larger version of this figure.

Supplementary Figure 1: Video still image (video time point 01:54 - 01:55) showing the left auricle (left atrial appendage). The arrow points to the inferomedial tip of the left auricle which is visible as a triangular structure at the superior left edge of the heart. In case the LAD is not clearly visualized, the tip of the left auricle can be used as a landmark for needle entry to pass a suture under the LAD. The entry point is $1 \mathrm{~mm}$ inferior and medial to the inferiormost point the tip of the left auricle reaches during dynamic movements of the heart (black arrow shows inferomedial tip of the left auricle). Please click here to download this figure.

\section{Discussion}

The method facilitates the operator to efficiently transplant a bioprinted patch by applying it to the epicardial surface of an infarcted mouse heart following permanent LAD ligation. In this feasibility-focused method, we are able to perform this procedure on eight mice per working day (including preparation of the room before and afterwards). A bioprinting run producing eight $1 \mathrm{~cm}^{2}$ patches in wells of six-well plates takes 2-3 hours (including preparation time before and after). We used the sterile inside of a surgical scalpel packet as the scoop for our patch, which is readily accessible and generally adds minimal cost, utilizing the natural adhesive properties of the alginate/gelatine hydrogel patch to drape the patch across the anterior infarcted surface of the heart. In our experience, the protocol for LAD ligation in mice is operator dependent and a lower mortality rate at 28 days can be achieved with experienced operators specialized in one model. Van den Borne et al. ${ }^{16}$ reported that C57BL6 mice present a $44 \%$ mortality following permanent LAD ligation at 28 days without the application of a patch, which is higher than the upper limit of $30 \%$ that we observed with the method. 
The intubation step is critical and in-and-of-itself can be a source of mortality for mice unless performed by a skilled operator. It is made difficult due to the tiny size of the trachea, which is why magnifying glasses are worn by the operator for this step. We use injected ketamine/xylazine as well as inhaled isofluorane for induction of anesthetic so that the mouse is deeply anesthetized at relatively low doses of each drug. Therefore, there is no risk for the mouse to wake up during this intubation step but the high mortality associated with high single-drug doses is avoided. Atropine was also given to counteract side effects such as bradycardia and hypersalivation. The use of a spotlight applied to the throat externally lights up the trachea internally so it is more visible and the vocal cords must be visualized opening and closing with the mouse's respiratory rate (usually $\sim 120$ breaths per minute). It is critical to position the mouse perfectly (which is why a hard surface is preferred rather than a warming mat underneath the mouse for this step) with the two incisor teeth held by a looped thread and the tongue retracted extremely gently with blunt forceps/pair of spatulas to open the mouth and visualize the trachea. Once the intubation is completed, the operator must be careful not to dislodge the tube in the transfer from the intubation area to the operating bed (which does have a heat mat underneath it to prevent hypothermia). When connecting the breathing tube to the ventilator apparatus, it is critical to stabilize the tube with one hand and connect the ventilator circuit with the other, so that there is minimal movement of the breathing tube such as pushing it more deeply into the trachea when connecting the ventilator segment of the tubing.

In this study, we used alginate $4 \%(\mathrm{w} / \mathrm{v}) /$ gelatine $8 \%(\mathrm{w} /$ v) in Dulbecco's Modified Eagle Medium (DMEM). Alginate/ gelatine hydrogels are known for their biocompatibility, low cost and biomechanical properties making them useful for
$3 D$ tissue engineering strategies ${ }^{23}$. These hydrogels can be crosslinked by mild gelation by adding calcium ions, which allows for viscosity to be altered. After bioprinting, we applied calcium chloride $\left(\mathrm{CaCl}_{2}\right) 2 \%(\mathrm{w} / \mathrm{v})$ in phosphatebuffered saline (PBS) onto patches and then cultured them in DMEM in six well plates for 7-14 days before transplanting them. This was the optimal window after patches containing cardiac cells started to beat in culture but before patches started to disintegrate. Whilst $\mathrm{CaCl}_{2}$ could be added regularly throughout the post-bioprinting phase to reduce patch disintegration, we found that the intrinsic viscosity of the hydrogel was sufficient for patches to maintain their structure up to transplantation with only one initial dose of $\mathrm{CaCl}_{2}$.

The method allowed for successful transplantation without sutures (which may damage the heart) or glue (which may block the interface between the patch and the heart). Future studies may confirm the hypothesis that sutureless and glueless transplantation does not negatively impact engraftment in mice as it is critical that the patch does not slip off the heart or interfere with the lungs. Other studies assessing engraftment of patches in permanent LAD ligation models with patch-based repair ${ }^{3}$ have measured engrafted area $\left(\mathrm{mm}^{2}\right)$ remaining with time $e^{24}$, the grafted patch thickness $(\mu \mathrm{m})$ remining with time $^{25}$, quantification of transplanted cells by polymerase chain reaction $(\mathrm{PCR})^{26}$ or bioluminescence photon emission flux of labelled live donor cells (a measure of photons emitted per second which can quantify labelled grafted cells surviving in living animals over time $)^{27}$. Future studies may use these methods to further evaluate whether sutureless and glueless transplantation affects patch engraftment (as well as structural and functional effects on the host myocardium). Nonetheless, macroscopically after 28 days in vivo in our immunocompetent mice, the anterior mediastinum 
presented variable fibrinous material and adhesions. The mechanism of patch-based cardiac regeneration may be from stimulation of host macrophage inflammatory responses ${ }^{19}$ or secreted immunological factors ${ }^{20}$ rather than numerical cell replenishment. If inflammation plays a positive role, the presence of foreign hydrogel material may be beneficial. Alternatively, to reduce the presence of foreign material it may be beneficial if the hydrogel component disintegrates over time. In fact, some approaches use biomaterials which support cells initially and then disintegrate, leaving only tissue 28,29 . Future studies to fully analyze patch engraftment and better understand the mechanisms behind patch-based cardiac regeneration may lead to optimized experimental designs before translation to human trials ${ }^{2}$.

Overall, this protocol is likely to be widely feasible and also suited to testing multiple groups of 3D bioprinted patches, for instance with different cellular contents. Future directions for this method include the bioprinting of patches containing advanced hydrogels not previously tested in vivo or testing the effects of different autologous or allogenic stem cellderived cells, for optimization before proceeding to large animal models.

\section{Disclosures}

None.

\section{FUNDING STATEMENT:}

Christopher D. Roche was supported by a Sir John Loewenthal Scholarship 2019 (University of Sydney), the Le Gros Legacy Fund New Zealand (PhD012019) and a Heart Research Australia PhD Scholarship (2019-02). Carmine Gentile was supported by a University of Sydney Kick-Start Grant, University of Sydney Chancellor's Doctoral Incentive Programme Grant, UTS Seed Funding, Catholic Archdiocese of Sydney Grant for Adult Stem Cell Research and a Sydney Medical School Foudation Cardiothoracic Surgery Research Grant.

\section{Acknowledgments}

With thanks to Natalie Johnston for the recording of the nonsurgical footage and all video editing.

\section{References}

1. Lund, L. H. et al. The registry of the International Society for Heart and Lung Transplantation: thirty-fourth adult heart transplantation report-2017; focus theme: allograft ischemic time. Journal of Heart and Lung Transplantation. 36 (10), 1037-1046 (2017)

2. Roche, C. D., Brereton, R. J. L., Ashton, A. W., Jackson, C., Gentile, C. Current challenges in threedimensional bioprinting heart tissues for cardiac surgery. European Journal of Cardio-Thoracic Surgery. 58 (3), 500-510 (2020).

3. Wang, H., Roche, C.D., Gentile, C. Omentum support for cardiac regeneration in ischaemic cardiomyopathy models: a systematic scoping review. European Journal of Cardio-Thoracic Surgery. ezaa205. Epub ahead of print (2020).

4. Mattapally, S. et al. Spheroids of cardiomyocytes derived from human-induced pluripotent stem cells improve recovery from myocardial injury in mice. American Journal of Physiology-Heart and Circulatory Physiology. 315 (2), H327-h339 (2018).

5. Gao, L. et al. Large cardiac muscle patches engineered from human induced-pluripotent stem cell-derived cardiac cells improve recovery from myocardial infarction in swine. Circulation. 137 (16), 1712-1730 (2018). 
6. Yang, B. et al. A net mold-based method of biomaterialfree three-dimensional cardiac tissue creation. Tissue Engineering Methods (Part C). 25 (4), 243-252 (2019).

7. Menasché, P. et al. Human embryonic stem cell-derived cardiac progenitors for severe heart failure treatment: first clinical case report. European Heart Journal. 36 (30), 2011-2017 (2015).

8. Menasché, P. et al. Transplantation of human embryonic stem cell-derived cardiovascular progenitors for severe ischemic left ventricular dysfunction. Journal of the American College of Cardiology. 71 (4), 429-438 (2018).

9. Beyersdorf, F. Three-dimensional bioprinting: new horizon for cardiac surgery. European Journal of CardioThoracic Surgery. 46 (3), 339-341 (2014).

10. Noor, N. et al. 3D printing of personalized thick and perfusable cardiac patches and hearts. Advanced Science. 6 (11), 1900344 (2019).

11. Maiullari, F. et al. A multi-cellular 3D bioprinting approach for vascularized heart tissue engineering based on HUVECs and iPSC-derived cardiomyocytes. Scientific Reports. 8 (1), 13532 (2018).

12. Zhang, Y. S. et al. Bioprinting 3D microfibrous scaffolds for engineering endothelialized myocardium and hearton-a-chip. Biomaterials. 110 45-59 (2016).

13. Chachques, J. C. et al. Myocardial assistance by grafting a new bioartificial upgraded myocardium (MAGNUM clinical trial): one year follow-up. Cell Transplant. 16 (9), 927-934 (2007).

14. Chachques, J. C. et al. Elastomeric cardiopatch scaffold for myocardial repair and ventricular support. European Journal of Cardio-Thoracic Surgery. 57 (3), 545-555 (2020).
15. Reichert, K. et al. Murine left anterior descending (LAD) coronary artery ligation: an improved and simplified model for myocardial infarction. Journal of Visualized Experiments. (122) (2017).

16. van den Borne, S. W. M. et al. Mouse strain determines the outcome of wound healing after myocardial infarction. Cardiovascular Research. 84 (2), 273-282 (2009).

17. Noguchi, R. et al. Development of a three-dimensional pre-vascularized scaffold-free contractile cardiac patch for treating heart disease. Journal of Heart and Lung Transplantation. 35 (1), 137-145 (2016).

18. Walker, R. L., Eggel, M. From mice to monkeys? Beyond orthodox approaches to the ethics of animal model choice. Animals. 10 (1), 77 (2020).

19. Vagnozzi, R. J. et al. An acute immune response underlies the benefit of cardiac stem-cell therapy. Nature. 577, 405-409 (2019).

20. Waters, R. et al. Stem cell-inspired secretome-rich injectable hydrogel to repair injured cardiac tissue. Acta Biomaterialia. (69), 95-106 (2018).

21. Cossu, G. et al. Lancet Commission: stem cells and regenerative medicine. Lancet. 391 (10123), 883-910 (2018).

22. Kawamura, M. et al. Enhanced therapeutic effects of human iPS cell derived-cardiomyocyte by combined cellsheets with omental flap technique in porcine ischemic cardiomyopathy model. Scientific Reports. 7 (1), 8824 (2017).

23. Lee, K. Y., Mooney, D. J. Alginate: properties and biomedical applications. Progress in Polymer Science. 37 (1), 106-126 (2012). 
24. Kainuma, S. et al. Cell-sheet therapy with omentopexy promotes arteriogenesis and improves coronary circulation physiology in failing heart. Molecular Therapy. 23 (2), 374-386 (2015).

25. Suzuki, R. et al. Omentopexy enhances graft function in myocardial cell sheet transplantation. Biochemical and Biophysical Research Communications. 387 (2), 353-359 (2009).

26. Zhou, Q., Zhou, J.Y., Zheng, Z., Zhang, H., Hu, S.S. A novel vascularized patch enhances cell survival and modifies ventricular remodeling in a rat myocardial infarction model. Journal of Thoracic and Cardiovascular Surgery. 140 (6), 1388-1396.e1383 (2010).

27. Lilyanna, S. et al. Cord lining-mesenchymal stem cells graft supplemented with an omental flap induces myocardial revascularization and ameliorates cardiac dysfunction in a rat model of chronic ischemic heart failure. Tissue Engineering (Part A). 19 (11-12), 1303-1315 (2013)

28. Miller, J. S. et al. Rapid casting of patterned vascular networks for perfusable engineered three-dimensional tissues. Nature Materials. 11 (9), 768-774 (2012).

29. Zhang, B. et al. Biodegradable scaffold with built-in vasculature for organ-on-a-chip engineering and direct surgical anastomosis. Nature Materials. 15 (6), 669-678 (2016). 